\title{
Propriedades químicas e mecânicas de filme bioativo de amido de mandioca com adição de extrato de jamelão (Syzygium cumini L.)
}

\author{
Chemical and mechanical properties of cassava starch \\ bioactive film with jambolan (Syzygium cumini L.) extract \\ addition
}

\section{Edinara Lacerda Queiroz ${ }^{1 *}$ (D), Geiza Suzart Araújo', Tamires Bastos Almeida1, Ernesto Acosta Martinez ${ }^{1}$ (D), Sílvia Maria Almeida de Souza ${ }^{1}$}

${ }^{1}$ Universidade Estadual de Feira de Santana (UEFS), Departamento de Tecnologia, Curso Engenharia de Alimentos, Feira de Santana/BA - Brasil

${ }^{*}$ Corresponding author: Edinara Lacerda Queiroz, Universidade Estadual de Feira de Santana (UEFS), Departamento de Tecnologia, Curso Engenharia de Alimentos, Av. Transnordestina s/n, Bairro Novo Horizonte, CEP: 44036-900, Feira de Santana/BA - Brasil, e-mail: edinaralacerdaq@gmail.com

Cite as: Queiroz, E. L., Araújo, G. S., Almeida, T. B., Martinez, E. A., \& Souza, S. M. A. (2021). Chemical and mechanical properties of cassava starch bioactive film with jambolan (Syzygium cumini L.) extract addition. Brazilian Journal of Food Technology, 24, e2020216. https://doi.org/10.1590/1981-6723.21620

\begin{abstract}
Resumo
O jamelão é uma fruta encontrada nas regiões Norte, Sudeste e Nordeste do Brasil. O extrato dos frutos apresenta atividade antioxidante, devido à presença de compostos bioativos, como carotenoides e compostos fenólicos. Este estudo objetivou elaborar filmes comestíveis, compostos por amido de mandioca como matriz principal, com a adição de extrato de jamelão. A polpa de jamelão foi concentrada em roto-evaporador para a obtenção do extrato aquoso. Os ensaios para a elaboração dos filmes foram realizados segundo um delineamento composto central $2^{2}$ com três repetições no ponto central, para avaliar o efeito das concentrações de glicerol $(7,95 \%$ a $22,05 \%)$ e de extrato de jamelão $(15,9$ a 44,1\%) sobre as propriedades mecânicas do filme (força na perfuração e deformação na ruptura) e sobre o teor de compostos fenólicos. As maiores concentrações do plastificante glicerol e do extrato de jamelão influenciaram positivamente nas propriedades mecânicas do filme, provocando diminuição da força na perfuração de 3,61 N para 2,55 N e aumento da deformação na ruptura de 1,44\% para 76,33\%. Os efeitos lineares e quadráticos das concentrações de glicerol e de extrato de jamelão e suas interações foram significativas sobre as respostas: força na perfuração e deformação na ruptura, no nível de $95 \%$ de confiança. A concentração de extrato de jamelão teve efeito significativo sobre o teor de compostos fenólicos, com coeficiente de determinação $R^{2}=0$,9782. A interação entre glicerol e extrato de jamelão auxilia na obtenção de filmes de amido de mandioca com boas propriedades mecânicas e bioativas.
\end{abstract}

Palavras-chave: Embalagem; Plastificante; Antioxidante; Fenóis; Força; Deformação; Comestível. 


\begin{abstract}
Jambolan is a fruit found in the north, southeast and northeast of Brazil. The fruit extract has high antioxidant activity, due to the presence of bioactive compounds, such as carotenoids and phenols. The study aimed to elaborate edible films from cassava starch as matrix and addition of jambolan extract. The jambolan aqueous extract was obtained by concentrating process using a rotary evaporator. The assays were carried out according to a $2^{2}$ full-factorial central composite design with three center point replicates, to assess the effects of glycerol (from $7.95 \%$ to $22.05 \%$ ) and jambolan extract (from $15.9 \%$ to $44.1 \%$ ) concentrations on the mechanical properties (force and deformation at the breaking point) and phenols content of the film. A reduction of the force from $3.61 \mathrm{~N}$ to $2.55 \mathrm{~N}$, and increase of puncture deformation from $1.44 \%$ to $76.33 \%$ were verified with the use of high contents of glycerol and jambolan extract. A statistical analysis of the results showed that the linear and quadratic effects of glycerol and jambolan extract concentrations and their interactions were significant on the force and puncture deformation at a $95 \%$ and $80 \%$ confidence level, respectively. The effect of jambolan extract concentration was significant on phenols content at a $95 \%$ confidence level with coefficient of determination $\left(R^{2}\right)$ 0.9782. The interactions between glycerol and jambolan extract facilitated the formation of cassava starch films with good mechanical and bioactive properties.
\end{abstract}

Keywords: Packing; Plasticizer; Antioxidant; Phenols; Force; Deformation; Edible.

\title{
1 Introdução
}

Atualmente, a tendência de uso de filmes comestíveis tem se ampliado devido às crescentes preocupações ambientais, à necessidade de reduzir a quantidade de resíduos e ao aumento da demanda dos consumidores por produtos alimentícios de alta qualidade (Zhang et al., 2018). As embalagens ativas têm desempenhado diversas funções em alimentos, como aquelas com controle de entrada de $\mathrm{O}_{2}$ para alimentos facilmente oxidáveis, auxiliando no aumento da vida de prateleira (Gaikwad et al., 2018), e outras com retenção de aditivos (Moraes et al., 2011), sequestro de etileno (Sapper \& Chiralt, 2018) e ação antimicrobiana (López et al., 2018). Ressalta-se o mais importante: os filmes fornecem uma barreira protetora para evitar a transferência de material entre os alimentos e o meio ambiente, melhorando sua estabilidade (Huntrakul et al., 2020).

Filmes comestíveis preparados a partir de fontes não convencionais de amido podem se tornar um substituto eficaz para filmes de amido comuns (Pająk et al., 2019). Trabalhos publicados relatam a produção de filmes ativos elaborados com amido de mandioca incorporado de chá verde e manjericão (MedinaJaramillo et al., 2017), cravo e canela (Kechichian et al., 2010), e com blendas de amido de mandioca e poli (butileno adipato cotereftalato) (PBAT) (Brandelero et al., 2013).

Os filmes de amido têm características físicas adequadas, pois são inodoros, incolores, sem sabor e impermeáveis ao oxigênio (Sadeghizadeh-Yazdi et al., 2019). Água e glicerol são os plastificantes mais comuns empregados nos bioplásticos de amido, e a principal vantagem do seu uso é o aumento da flexibilidade do filme (Moro et al., 2017). A adição de plastificante deve ser feita com parcimônia, uma vez que a presença de moléculas com ação plastificante na matriz polimérica não altera somente as propriedades mecânicas, mas interfere na molhabilidade e na permeação desses filmes (Assis \& Britto, 2014).

O jamelão (Syzygium cumini L.) é uma fruta originária da Índia, pertencente à família das mirtáceas, e pode ser encontrada nas regiões Norte, Sudeste e Nordeste do Brasil (Leão et al., 2014). O extrato dos frutos do jamelão demonstra alta atividade antioxidante, a qual se deve à presença de compostos bioativos, como carotenoides e compostos fenólicos (Faria et al., 2011).

Filmes à base de amido, com propriedade antioxidante e protetora, podem ser aplicados como embalagem para diversos alimentos, frutas e hortaliças minimamente processadas, que têm alta perecibilidade, aumentando sua vida de prateleira e reduzindo perdas (Assis \& Britto, 2014). Extratos naturais, como extratos de plantas, frutas e óleos essenciais, foram reconhecidos como potenciais agentes antioxidantes e antimicrobianos em filmes (Halonen et al., 2020). 
A incorporação de extrato de semente de toranja e chá verde em filmes demonstrou resultados bem sucedidos, sendo ativos como antioxidantes e contra diferentes patógenos (por exemplo, Escherichia coli e Listeria spp.) (Wang \& Rhim, 2016; Wrona et al., 2017). A incorporação de eugenol, composto fenólico com alta atividade antimicrobiana e antioxidante, lecitina e ácido oleico em filme de amido foi altamente eficiente na prevenção da oxidação do óleo de girassol (Talón et al., 2019). Filmes comestíveis à base de amido de mandioca, com adição de extrato de folhas de alecrim (Bonilla et al., 2013), mostraram um aumento da concentração de tocoferóis e da atividade antioxidante, demonstrando o grande potencial deste extrato como agente antioxidante natural para a preservação de alimentos.

O presente trabalho objetiva elaborar filmes comestíveis, compostos por amido de mandioca como matriz principal, com a adição de glicerol e do extrato dos frutos do jamelão, visando ao aproveitamento da capacidade antioxidante do extrato.

\section{Materiais e métodos}

\subsection{Matérias-primas}

Para a elaboração do filme, foram utilizados o amido de mandioca (Manihot esculenta Crantz) de grau alimentício, como matriz principal; água, como solvente; glicerol, como agente plastificante, e o extrato do jamelão, como aditivo com propriedade antioxidante. $\mathrm{O}$ amido de mandioca (Marca Loanda) foi obtido no comércio local na cidade de Feira de Santana-Bahia. Os frutos do jamelão foram colhidos no Campus da Universidade Estadual de Feira de Santana (UEFS) e na zona rural da região, no período de dezembro a março de 2017. O reagente e os equipamentos utilizados foram: glicerina P.A. ACS (Marca Synth), estufa (FANEM Modelo SL-102/100), agitador magnético (Fisatom-modelo 752A), Evaporador rotativo 1LT digital e Banho-maria analógico (Marca Fisatom).

\subsection{Obtenção do extrato de jamelão}

Após higienização, a polpa de jamelão foi diluída em água destilada, utilizando a proporção de $1: 3\left(\mathrm{~m} \cdot \mathrm{m}^{-1}\right)$ fruta:água, de acordo com a metodologia proposta por Roesler et al. (2007), com adaptações. Para a obtenção do extrato, utilizou-se a mesma proporção de fruta:solvente proposta pelos autores; no entanto, utilizou-se a água como solução extratora em vez do etanol, obtendo-se assim um extrato aquoso de jamelão. Esta mistura foi homogeneizada em mixer (1 min), filtrada e concentrada em rota-evaporador (Fisatom, Modelo 826T) a $40{ }^{\circ} \mathrm{C}$, obtendo-se assim o extrato bruto, que foi adicionado ao filme.

\subsection{Elaboração do filme}

Os filmes comestíveis, à base de amido de mandioca $(4 \mathrm{~g})$, foram elaborados de acordo com a metodologia casting, proposta por Shimazu et al. (2007), com adaptações. As soluções filmogênicas foram aquecidas de 30 a $95^{\circ} \mathrm{C}$, mantidas a $95^{\circ} \mathrm{C}$ por 10 min e resfriadas até $50^{\circ} \mathrm{C}$, quando o extrato foi adicionado. Em seguida, $50 \mathrm{~g}$ de cada solução filmogênica foi espalhada em placa de acrílico $(15 \times 21 \mathrm{~cm})$. O material foi seco em estufa com circulação $(2 \mathrm{~m} / \mathrm{s})$ e renovação de ar a $40{ }^{\circ} \mathrm{C}$ por $24 \mathrm{~h}$. Antes das análises, os filmes foram acondicionados em dessecadores, sob ambiente artificial com nitrato de magnésio (umidade relativa de 57\%) e temperatura ambiente $\left(30^{\circ} \mathrm{C}\right)$, conforme os métodos e normas da American Society for Testing and Materials (2001). Todas as análises foram realizadas em triplicata.

As condições experimentais iniciais de processo foram baseadas em dados da literatura referentes ao processo casting, na elaboração e caracterização de filmes comestíveis (Rigo, 2006; Vicentini, 2003; Shimazu et al., 2007; Berthan, 2008). 


\subsection{Planejamento fatorial para elaboração dos filmes}

O Delineamento Central Composto Rotacional $2^{2}$, com oito ensaios e três repetições no ponto central, foi utilizado para avaliar os efeitos das concentrações de plastificante $\left(\mathrm{X}_{1}\right)$ e de extrato de jamelão $\left(\mathrm{X}_{2}\right)$ sobre a ação antioxidante do extrato, e as propriedades mecânicas do filme (força na perfuração e deformação na ruptura).

Os valores reais e codificados das variáveis independentes que compõem cada solução filmogênica do delineamento experimental são mostrados na Tabela 1.

Tabela 1. Valores reais e codificados do planejamento experimental $2^{2}$ para avaliar os efeitos das concentrações de plastificante $\left(X_{1}\right)$ e de extrato de jamelão $\left(X_{2}\right)$ sobre a ação antioxidante do extrato, e as propriedades mecânicas do filme.

\begin{tabular}{|c|c|c|}
\hline \multirow[b]{2}{*}{ Ensaio } & $\mathbf{X}_{1}$ & \multirow[t]{2}{*}{$\mathbf{X}_{2}$} \\
\hline & Glicerol $(\%)^{a}$ & \\
\hline 1 & $10(-1)$ & $20(-1)$ \\
\hline 2 & $10(-1)$ & $40(1)$ \\
\hline 3 & $20(1)$ & $20(-1)$ \\
\hline 4 & $20(1)$ & $40(1)$ \\
\hline 5 & $7,95(-1,41)$ & $30(0)$ \\
\hline 6 & $22,05(1,41)$ & $30(0)$ \\
\hline 7 & $15(0)$ & $15,9(-1,41)$ \\
\hline 8 & $15(0)$ & $44,01(1,41)$ \\
\hline 9 & $15(0)$ & $30(0)$ \\
\hline 10 & $15(0)$ & $30(0)$ \\
\hline 11 & $15(0)$ & $30(0)$ \\
\hline
\end{tabular}

${ }^{\mathrm{a}} \mathrm{g} / 100 \mathrm{~g}$ de amido de mandioca.

Os dados foram tratados estatisticamente pelo método de análise de variância (ANOVA) e da metodologia de Superfície de Resposta (RSM), utilizando o software Statistica 7.0. Os resultados foram avaliados estatisticamente e as diferenças foram consideradas significativas no nível de $95 \%$ de probabilidade $(p<$ $0,05)$.

\subsection{Análise de compostos fenólicos}

O extrato bruto e o filme foram caracterizados físico-quimicamente, com análise do teor de compostos fenólicos, por meio da metodologia proposta por Roesler et al. (2007), com adaptações, que utiliza o método Folin Ciocalteau. Para a determinação do teor de fenóis totais, $1 \mathrm{~g}$ da amostra foi diluído em $9 \mathrm{~mL}$ de solução extratora e, posteriormente, foram adicionados, num tubo de ensaio: uma alíquota de $0,5 \mathrm{~mL}$ da amostra diluída; 2,5 mL do reagente Folin Ciocalteau (Marca Synth), na proporção 1:10, e $2 \mathrm{~mL}$ de carbonato de sódio a 7,5\%. A solução foi incubada a $50{ }^{\circ} \mathrm{C}$ por $15 \mathrm{~min}$, após ser resfriada à temperatura ambiente, sendo então feita a leitura de absorbância a $760 \mathrm{~nm}$, em espectrofotômetro UV/Vis (Femto Modelo 600 Plus). A quantificação de fenóis foi feita através de uma curva padrão de ácido gálico e o resultado expresso em equivalente de ácido gálico-GAE (mg GAE/g de amostra). 


\subsection{Análise de propriedades mecânicas}

As análises de propriedades mecânicas de perfuração do filme, com determinação de força e deformação, foram realizadas de acordo com a metodologia de Gontard et al. (1993), em texturômetro (Stable Micro Systems, Inglaterra). Os filmes foram fixados em célula circular de encaixe com $3 \mathrm{~cm}$ de diâmetro e perfurados com uma sonda metálica cilíndrica com diâmetro de $3 \mathrm{~mm}$, a uma velocidade de deslocamento de $1 \mathrm{~mm} / \mathrm{s}$. A espessura dos filmes foi determinada de acordo com a metodologia proposta por Rigo (2006), segundo a qual fixou-se o peso de $50 \mathrm{~g}$ de solução filmogênica depositada nas placas. Após a secagem e armazenagem em umidade controlada, a espessura foi mensurada em um micrômetro digital $( \pm 0,001 \mathrm{~mm})$ em oito pontos diferentes no perímetro de seis amostras de filme.

\section{Resultados e discussão}

\subsection{Caracterização do extrato de jamelão}

O extrato aquoso de jamelão apresenta alta concentração de compostos fenólicos $(585,48 \mathrm{mg} \mathrm{GAE} / 100 \mathrm{~g})$, quando comparado aos extratos de outras frutas, como o extrato aquoso do resíduo de polpa de acerola, que apresentou teor de fenóis de 247,62 mg GAE/100 g (Sousa et al., 2011). As frutas utilizadas para a obtenção do extrato se encontravam no estágio de maturação chamado de [meio maduro] observando-se que quanto maior o grau de maturação, menor o teor de compostos fenólicos (Veber et al., 2015). O teor de fenóis no extrato aquoso foi $63 \%$ superior ao do extrato de jamelão hidroetanólico $50 \%$ (v/v) obtido por Veber et al. (2015). A composição química do extrato depende da espécie, das condições ambientais e, também, do estágio de maturação do fruto (Vendramini \& Trugo, 2000).

\subsection{Caracterização dos filmes}

Os filmes elaborados com amido de mandioca, com diferentes concentrações de glicerol e extrato de jamelão, se apresentaram, em geral, manuseáveis, uniformes, transparentes e com bom aspecto tátil. Todas as formulações apresentaram coloração levemente rosada, característica do extrato adicionado. A cor se tornava mais intensa quanto maior a concentração de extrato na solução filmogênica. A espessura média dos filmes variou de 0,032 a $0,083 \mathrm{~mm}$. Os resultados experimentais para os parâmetros - força na perfuração, deformação na ruptura e teor de compostos fenólicos nos filmes - são mostrados na Tabela 2.

Tabela 2. Valores de força na perfuração, deformação na ruptura e teor de fenóis nos ensaios dos filmes incorporados de glicerol e extrato concentrado de jamelão.

\begin{tabular}{|c|c|c|c|c|c|}
\hline \multirow[b]{2}{*}{ Ensaio } & \multicolumn{2}{|c|}{ Variáveis independentes } & \multicolumn{3}{|c|}{ Variáveis dependentes } \\
\hline & Glicerol $^{\mathrm{a}}(\%)$ & Extrato $^{a}(\%)$ & Força $(\mathbf{N})$ & $\begin{array}{c}\text { Deformação na } \\
\text { ruptura }(\%)\end{array}$ & Fenóis (mg GAE/g de filme) \\
\hline 1 & 10 & 20 & $3,616 \pm 0,04$ & $19,280 \pm 0,40$ & $2,01 \pm 0,00$ \\
\hline 2 & 10 & 40 & $2,941 \pm 0,09$ & $25,491 \pm 0,59$ & $2,69 \pm 0,01$ \\
\hline 3 & 20 & 20 & $2,680 \pm 0,03$ & $43,520 \pm 0,34$ & $2,08 \pm 0,02$ \\
\hline 4 & 20 & 40 & $2,562 \pm 0,06$ & $76,332 \pm 0,17$ & $2,65 \pm 0,02$ \\
\hline 5 & 7,95 & 30 & $3,064 \pm 0,05$ & $21,042 \pm 0,41$ & $2,19 \pm 0,01$ \\
\hline 6 & 22,05 & 30 & $2,712 \pm 0,06$ & $58,558 \pm 0,35$ & $2,30 \pm 0,02$ \\
\hline 7 & 15 & 15,9 & $2,842 \pm 0,03$ & $1,447 \pm 0,30$ & $1,97 \pm 0,00$ \\
\hline 8 & 15 & 44,01 & $2,555 \pm 0,09$ & $28,414 \pm 0,28$ & $2,99 \pm 0,01$ \\
\hline 9 & 15 & 30 & $3,383 \pm 0,06$ & $17,746 \pm 0,16$ & $2,14 \pm 0,00$ \\
\hline 10 & 15 & 30 & $3,383 \pm 0,09$ & $18,853 \pm 0,29$ & $2,22 \pm 0,01$ \\
\hline 11 & 15 & 30 & $3,345 \pm 0,07$ & $19,008 \pm 0,26$ & $2,21 \pm 0,01$ \\
\hline
\end{tabular}

${ }^{\mathrm{a}}$ Os teores adicionados foram calculados em $\mathrm{g} / 100 \mathrm{~g}$ de amido. 
Os filmes com menores concentrações de plastificante apresentaram maior rigidez e alguns se tornaram quebradiços. Com relação ao extrato de jamelão, quanto maior sua concentração, maior o teor de compostos fenólicos e melhor a elasticidade do filme. Tal fato poderia estar relacionado à interação dos açúcares presentes no extrato da fruta, os quais, junto ao glicerol, atuaram como um plastificante extra.

A seleção do plastificante é extremamente importante, uma vez que este garante a flexibilidade da matriz polimérica e deve ser adequadamente compatível com o polímero utilizado (Rocha et al., 2014). Os plastificantes são necessários por interagir na matriz polimérica, através de ligações de hidrogênio ao longo das cadeias poliméricas na região amorfa do amido, diminuindo as forças intermoleculares e aumentado o volume livre e a flexibilidade da cadeia (Moro et al., 2017). Verifica-se, como consequência, a diminuição de possíveis descontinuidades e de zonas quebradiças.

A força $(\mathrm{N})$ é classificada como a resistência do material à perfuração e, nos filmes, este parâmetro variou de 2,55 a 3,61 N. Pode-se constatar que maiores valores de força são obtidos nas condições de teor reduzido de extrato de jamelão (20\%) e menor teor de glicerol $(10 \%)$. A força na perfuração tem seus valores mínimos, $2,55 \mathrm{~N}$ e $2,56 \mathrm{~N}$, em concentrações elevadas de extrato de jamelão $(44,01 \%)$ e glicerol $(20 \%)$, respectivamente. A incorporação de plastificantes como o glicerol interfere na interação entre as cadeias de polímeros e na formação do produto, alterando as propriedades físicas do filme biodegradável (Brito, 2019).

O mesmo efeito de redução da força com o aumento na concentração do plastificante foi verificado em diversos trabalhos disponíveis na literatura, nos mais diversos períodos. Mali \& Grossmann (2003), na elaboração de um filme com $4 \%$ de amido de inhame e glicerol como agente plastificante, observaram que um aumento da concentração de glicerol de 1,3 para $2 \%$ causou a redução da força na perfuração de $14,6 \mathrm{~N}$ para 12,09 N. Farias et al. (2012), na elaboração de filmes com 4\% de amido de mandioca adicionado de glicerol e polpa de acerola, observaram que o aumento na concentração de glicerol de 20 para $40 \%$ ocasionou a diminuição na força de 12,3 N para 6,7 N. Esses autores também verificaram comportamento similar de redução na força com o aumento no teor de polpa de acerola.

A deformação na ruptura dos filmes variou de 1,44 a 76,33\%. Observou-se a capacidade do plastificante e do extrato em aumentar a deformação na ruptura dos filmes. Comportamentos similares foram relatados por Cunha (2017), acerca do filme de amido de mandioca adicionado de extrato de própolis $(28,39 \%)$; Souza et al. (2012a), com o filme de amido de mandioca adicionado de polpas de manga e acerola $(72,45 \%)$; Rodríguez et al. (2006), com o filme de amido de batata com surfactante (31,5\%), e Souza et al. (2012b), com o filme à base de proteínas miofibrilares de carne bovina (17,94\%), com a utilização de glicerol como agente plastificante em diferentes concentrações ( 0 a $75 \%$ ).

De acordo com Mali et al. (2010), os plastificantes aumentam a mobilidade das cadeias dos polímeros, com diminuição de descontinuidades e zonas quebradiças, o que se reflete num decréscimo da força na perfuração e no aumento da deformação na ruptura dos filmes. Assim, ocorre melhora na flexibilidade dos filmes formados e aumento do alongamento ou da deformação, devido à redução das interações entre as cadeias do amido (Galdeano et al., 2009). Geralmente, estruturas com maior grau de cristalinidade são mais resistentes mecanicamente, pois há um aumento na resistência ao escoamento e na dureza do material (Canevarolo et al., 2014).

$\mathrm{Na}$ análise de compostos fenólicos, pode-se verificar que o filme com maior teor $(2,99 \mathrm{mg} \mathrm{GAE} / \mathrm{g}$ de filme) é aquele incorporado com maior concentração de extrato aquoso de jamelão (44,1\%). Resultados similares foram reportados na literatura para filmes formulados com extrato de própolis. Cunha (2017), com o filme de amido adicionado de 30 e $60 \%$ de extrato etanólico de própolis, obteve teor de fenóis de 4,183 e $5,525 \mathrm{mg} \mathrm{GAE} / \mathrm{g}$ de filme, respectivamente. Bodini (2011) relatou teor de 8,8 $\mathrm{mgGAE} / \mathrm{g}$ de filme para formulação elaborada com gelatina como matriz principal e adição de extrato etanólico de própolis. É importante ressaltar que extratos de frutas e vegetais possuem excelente atividade antioxidante, e podem retardar a oxidação lipídica e melhorar a qualidade e a vida de prateleira de vários sistemas alimentares (Eça et al., 2014). 


\subsection{Análise Estatística}

A partir da análise de variância (ANOVA), foi possível estabelecer a significância da regressão e da falta de ajuste em relação a 95\% de confiança $(p \geq 0,05)$, através do teste $\mathrm{F}$. Os modelos para as variáveis dependentes, força e teor de compostos fenólicos, apresentaram regressões significativas, falta de ajustes adequados e valores satisfatórios de $\mathrm{R}^{2}(0,9565$ e 0,9782 , respectivamente). A variável resposta, deformação na ruptura, apresentou o modelo com menor coeficiente de determinação $\left[R^{2}(0,9036)\right.$ e Fcalculado=13,964], explicando parte das variações encontradas, mas ainda assim, todos os valores das regressões foram significativos, apresentando Fcalculado $>$ Ftabelado. Deste modo, pode-se afirmar que os modelos apresentaram significância estatística e que os mesmos são preditivos. Os modelos matemáticos, eliminando os fatores não significativos, que descrevem as respostas da força $(\mathrm{N})$, da deformação na ruptura (\%) e do teor de fenóis (mg GAE/g) do filme de amido adicionado de glicerol e de extrato de jamelão, são apresentados pelas Equações 1, 2 e 3, respectivamente.

$$
\begin{aligned}
& y_{2}=3,37-0,19 x_{1}-0,11 x_{2}-0,22 x_{1}^{2}-0,31 x_{2}^{2}+0,064 x_{1} x_{2} \quad \mathrm{R}^{2}=0,9565 \\
& y_{3}=17,73+16,02 x_{1}+9,64 x_{2}+15,08 x_{1}^{2}+2,64 x_{2}^{2}+6,65 x_{1} x_{2} \quad \mathrm{R}^{2}=0,9036 \\
& y_{4}=2,19+0,34 x_{2}+0,14 x_{2}^{2} \mathrm{R}^{2}=0,9782
\end{aligned}
$$

Em que: $x_{1}$ : Glicerol; $x_{2}$ : Extrato de jamelão; $y_{1}$ : força $(\mathrm{N}) ; y_{2}$ : deformação na ruptura $(\%) ; y_{3}$ : teor de fenóis (mgGAE/g de filme).

(A)

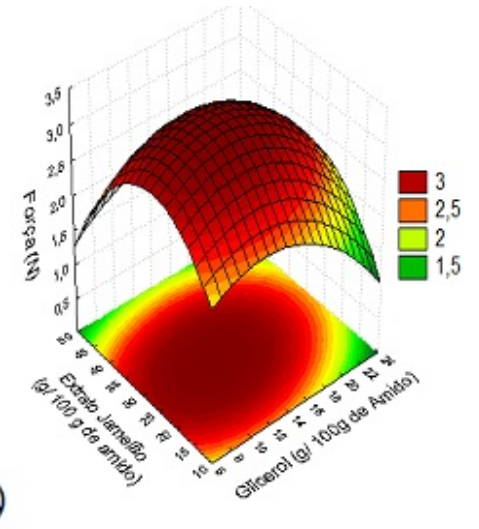

(B)

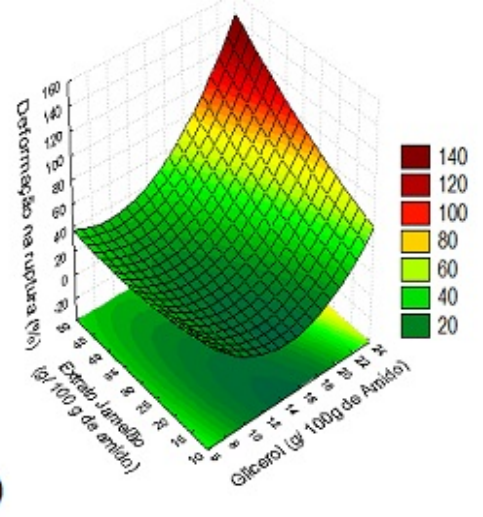

(C)

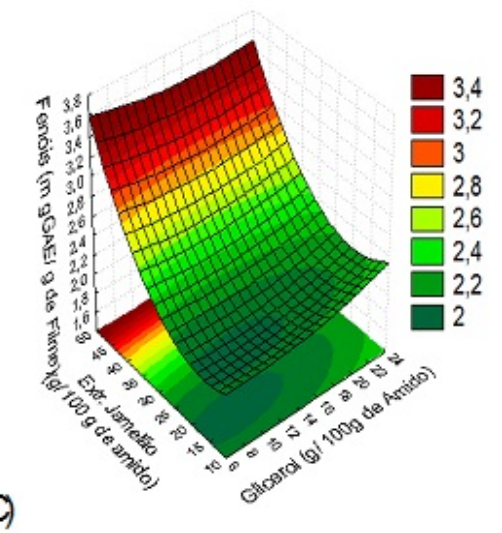

Figura 1. Superfícies de resposta e curvas de contorno descritas pelo modelo proposto para as respostas força na perfuração (A), deformação na ruptura (B) e teor de fenóis (C), em função das concentrações de glicerol e extrato de jamelão. 
De acordo com os gráficos da superfície de resposta e das curvas de contorno (Figura 1), pode-se verificar que a força na perfuração (Figura 1A) foi influenciada pelos efeitos lineares, quadráticos e suas interações do teor de glicerol e de extrato de jamelão, variando de 2,55 a 3,36 N. O menor valor de força do filme de jamelão se encontra na faixa de maiores concentrações de glicerol (15\% a 20\%) e maiores teores de extrato de jamelão (40\% a 44,01\%). Tal comportamento demonstra que à medida que aumenta os teores de plastificante e de extrato há uma redução na força de ruptura dos filmes. Por outro lado, foi verificado que a redução nos teores de plastificante e de extrato gerou um aumento na força de rompimento dos filmes. Entretanto, a força na ruptura teve aumento até alcançar o maior valor médio $(3,36 \mathrm{~N})$, com níveis intermediários de glicerol e extrato de jamelão (7,95\% e 30\%, respectivamente), e depois houve uma diminuição com o aumento da adição do teor de extrato. Esta redução, que foi discutida exaustivamente por vários autores (Souza et al., 2012a; Sobral et al., 2001; Gontard et al., 1993), tem relação com a função do plastificante, que ocorre, provavelmente, devido ao rompimento das ligações de hidrogênio intermoleculares entre as cadeias poliméricas de amido e o plastificante, com a adição contínua do extrato de jamelão, provocando, assim, uma possível desestabilidade na matriz polimérica com o aumento do grau de interação.

Este comportamento se repete para a resposta deformação na ruptura (Figura 1B). As variáveis independentes de extrato de jamelão - linear e quadrática $(p<0,05)$, glicerol linear $(p<0,05)$ e a interação $(p<0,05)$ - foram significativas sobre a resposta deformação. Observou-se que o maior valor $(76,33 \%)$ se encontra no filme com concentrações elevadas de glicerol (20\%) e extrato de jamelão (40\%). Um aumento nos teores das variáveis independentes, glicerol e extrato na solução filmogênica, bem como suas interações, favoreceu a deformação na ruptura do filme, com variação linear em sentido positivo e $p<0,05$. A ação do plastificante de aumentar a deformação dos filmes já é bastante conhecida: ele age entre as cadeias poliméricas, reduzindo interações, aumentando a mobilidade e, consequentemente, tornando os filmes mais extensíveis. Tal comportamento foi verificado no modelo e é observado na Figura 1B.

Os amidos podem formar filmes comestíveis com resistência mecânica e podem ser usados para revestir alimentos e prevenir a perda de água, além de proteger contra efeitos adversos (Dash et al., 2019). No entanto, filmes de amido puro mostram fragilidade e propriedades mecânicas pobres, e, desse modo, os plastificantes são necessários para melhorar suas características de flexibilidade (Li et al., 2020). Assim como observado no presente trabalho, outros autores também reportaram resultados semelhantes sobre o aumento da deformação na ruptura com o aumento da concentração de glicerol. Rocha et al. (2014) relataram que, em filmes de amido de mandioca, os valores de deformação na perfuração $(31,9 \%)$ ocorreram com teor de glicerol mais alto (47\%). Moro et al. (2017) verificaram que, em filmes de amido reforçado com casca de maracujá e concentração de glicerol entre 64 e $80 \%$, a deformação na ruptura variou de $9,5 \%$ a 39,9\%, sendo a ocorrência dos maiores valores em altas concentrações de plastificante.

A adição de plastificantes tem a função de melhorar a maleabilidade dos filmes, manter a integridade e prevenir poros e rachaduras na matriz polimérica (Sessini et al., 2016). Dentre os plastificantes utilizados, o poliol glicerol se destaca em função de a molécula possuir três grupos de hidroxila e peso molecular (PM) baixo, o que lhe confere a característica de formar ligações de hidrogênio intermoleculares, responsáveis por sua solubilidade em água e sua natureza higroscópica. Ademais, o poliol glicerol, ao ser adicionado a materiais poliméricos, modifica a organização espacial, diminui as forças intermoleculares atrativas e, dessa forma, aumenta o volume livre e a mobilidade da cadeia polimérica (Souza et al., 2012b).

O jamelão é uma fruta pequena, de cor roxa a preta, a qual é atribuída à presença de altas quantidades de antocianinas (Sari et al., 2012). O teor de fenóis dos filmes variou de 1,97 e 2,99 mg GAE/g de filme. A adição de extrato de jamelão foi significativa, com efeito positivo sobre a resposta teor de fenóis, tanto linear como quadrática, com valores de $p<0,05$.

Extratos de frutas têm grande aplicação na indústria, desde alimentos e produtos farmacêuticos, até a indústria de embalagens ativas, sendo incorporados como aditivos naturais, conferindo características funcionais às mesmas (Pastor et al., 2011). De acordo com os gráficos da superfície de resposta (Figura 1C), 
verificou-se que os filmes com maiores teores de compostos fenólicos (2,69 e 2,99 mg GAE/g de filme) são aqueles incorporados com maiores concentrações de extrato de jamelão (40\% e 44,1\%). Assim, observou-se que, com o aumento de extrato de jamelão de $15,9 \%$ para 44,01\%, o teor de fenóis aumenta de 1,97 para 2,99 $\mathrm{mg} \mathrm{GAE} / \mathrm{g}$ de filme. Para esta resposta, foi demonstrado comportamento linear em sentido positivo; logo, há um aumento do teor de fenóis, conforme mais elevada for a concentração de extrato adicionado na solução filmogênica. A variável glicerol linear e quadrática, assim como a interação com extrato de jamelão, não foram significativas $(p>0,05)$ sobre a resposta teor de fenóis.

\section{Conclusões}

As propriedades mecânicas dos filmes de amido foram diretamente influenciadas pelas concentrações de plastificante e de extrato de jamelão na solução filmogênica. Altas concentrações de glicerol e de extrato conferiram maior manuseabilidade, menor força e maior deformação na ruptura aos filmes. O extrato de jamelão apresentou alto teor de compostos fenólicos e, quanto maior sua adição às soluções filmogênicas, maior foi o teor destes compostos nos filmes.

Os filmes de amido de mandioca com adição de extrato de jamelão são uma alternativa promissora no mercado de embalagens bioativas, conforme observado neste estudo. Sob esta perspectiva, estudos aprofundados serão necessários para o uso e a conservação de alimentos suscetíveis à oxidação, aumentando a vida de prateleira e evitando perdas na cadeia produtiva.

\section{Agradecimentos}

Ao Conselho Nacional de Desenvolvimento Científico e Tecnológico (CNPq), pelo apoio financeiro e a Universidade Estadual de Feira de Santana (UEFS).

\section{Referências}

American Society for Testing and Materials - ASTM. (2001). Standard test methods for tensile properties of thin plastics sheeting (ASTM D 882-00). Philadelphia: ASTM.

Assis, O. B. G., \& Britto, D. (2014). Coberturas comestíveis protetoras em frutas: fundamentos e aplicações. Brazilian Journal of Food Technology, 17(2), 87-97. http://dx.doi.org/10.1590/bjft.2014.019

Berthan, L. C. (2008). Desenvolvimento e caracterização de biofilmes ativos à base de polímeros de fontes renováveis e sua aplicação no acondicionamento de pães de forma (Tese de doutorado). Universidade Estadual de Campinas, Campinas.

Bodini, R. B. (2011). Desenvolvimento de materiais poliméricos a base de gelatina e própolis (Dissertação de mestrado). Universidade de São Paulo, Pirassununga.

Bonilla, J., Talón, E., Atarés, L., Vargas, M., \& Chiralt, A. (2013). Effect of the incorporation of antioxidants on physicochemical and antioxidant properties of wheat starch-chitosan films. Journal of Food Engineering, 118(3), 271-278. http://dx.doi.org/10.1016/j.jfoodeng.2013.04.008

Brandelero, R. P. H., Grossmann, M. V., \& Yamashita, F. (2013). Hydrophilicity of starch and poly(butylene adipate-coterephthalate) (PBAT) films containing tween 80 and soybean oil. Polímeros: Ciência e Tecnologia, 23, 270.

Brito, J. H. (2019). Produção e caracterização estrutural, morfológica e térmica de filmes biodegradáveis utilizando amido de caroço de abacate (Persea americana Mill) e bagaço de mandioca (Manihot esculenta Crantz) (Dissertação de mestrado). Universidade Estadual de Ponta Grossa, Ponta Grossa.

Canevarolo, S. V., Bernardo, F. O. C., Hincapie, J. A. Z., Elias, M. B., \& Silva, J. (2014). Structural characterization of bioriented blown films of polypropylene/polyethylene blends. AIP Conference Proceedings, 1593, 650-653. http://dx.doi.org/10.1063/1.4873863

Cunha, G. F. (2017). Biofilmes à base de amido incorporados com extrato etanólico de própolis (Dissertação de mestrado). Instituto Federal De Educação, Ciência e Tecnologia Goiano, Rio Verde. Retrieved in 2021, September 1, from https://sistemas.ifgoiano.edu.br/sgcursos/uploads/anexos_11/2017-10-19-09-50-32Disserta\%C3\%A7\%C3\%A3o\%20Giana.pdf

Dash, K. K., Ali, N. A., Das, D., \& Mohanta, D. (2019). Thorough evaluation of sweet potato starch and lemon-waste pectin based-edible films with nano-titania inclusions for food packaging applications. International Journal of Biological Macromolecules, 139, 449-458. PMid:31374275. http://dx.doi.org/10.1016/j.ijbiomac.2019.07.193

Eça, K. S., Sartori, T., \& Menegalli, F. C. (2014). Films and edible coatings containing antioxidants - a review. (2014). Brazilian Journal of Food Technology, 17(2), 98-112. http://dx.doi.org/10.1590/bjft.2014.017 
Faria, A. F., Marques, M. C., \& Mercadante, A. Z. (2011). Identification of bioactive compounds from jambolão (Syzygium cumini) and antioxidant capacity evaluation in different pH conditions. Food Chemistry, 126(4), 1571-1578. PMid:25213929. http://dx.doi.org/10.1016/j.foodchem.2010.12.007

Farias, M. G., Fakhouri, F. M., Carvalho, C. W. P., \& Ascheri, J. L. R. (2012). Caracterização físico-química de filmes comestíveis de amido adicionado de acerola (Malphigia emarginata D.C.). Quimica Nova, 35(3), 546-552. http://dx.doi.org/10.1590/S0100-40422012000300020

Gaikwad, K. K., Singh, S., \& Lee, Y. S. (2018). Oxygen scavenging films in food packaging. Environmental Chemistry Letters, 16(2), 523-538. http://dx.doi.org/10.1007/s10311-018-0705-z

Galdeano, M. C., Mali, S., Grossmann, M. V. E., Yamashita, F., \& Garcia, M. A. (2009). Effects of plasticizers on the properties of oat starch films. Materials Science and Engineering, 29(2), 532-538. http://dx.doi.org/10.1016/j.msec.2008.09.034

Gontard, N., Guilbert, S., \& Cuq, J. L. (1993). Water and glycerol as plasticizers affect mechanical and water vapor barrier properties of an edible wheat gluten film. Journal of Food Science, 58(1), 206-211. http://dx.doi.org/10.1111/j.13652621.1993.tb03246.x

Halonen, N., Pálvölgyi, P. S., Bassani, A., Fiorentini, C., Nair, R., Spigno, G., \& Kordas, K. (2020). Bio-based smart material for food packaging and sensors: a review. Front. Mater, 7, 82. http://dx.doi.org/10.3389/fmats.2020.00082

Huntrakul, K., Yoksan, R., Sane, A., \& Harnkarnsujarit, N. (2020). Effects of pea protein on properties of cassava starch edible films produced by blown-film extrusion for oil packaging. Food Packaging and Shelf Life, 24, 100480.

http://dx.doi.org/10.1016/j.fpsl.2020.100480

Kechichian, V., Ditchfield, C., Veiga-Santos, P., \& Tadini, C. C. (2010). Natural antimicrobial ingredients incorporated in biodegradable films based on cassava starch. Lebensmittel-Wissenschaft + Technologie, 43(7), 1088-1094. http://dx.doi.org/10.1016/j.Iwt.2010.02.014

Leão, L. A. C., Gabardo, M. C. L., \& Gomara, F. L. (2014). Estudo fitoquímico do guapê, Syzygium cumini (L.) Skeels. Acta Biológica Paranaense, 43(1-2), 41-52.

Li, S., Ma, Y., Ji, T., Sameen, D. E., Ahmed, S., Qin, W., Dai, J., Li, S., \& Liu, Y. (2020). Cassava starch/carboxymethylcellulose edible films embedded with lactic acid bacteria to extend the shelf life of banana. Carbohydrate Polymers, 248, 116805. PMid:32919539. http://dx.doi.org/10.1016/j.carbpol.2020.116805

López, O. V., Castillo, L. A., Farenzena, S., Pintos, E., Rodríguez, M. S., García, M. A., \& Villar, M. A. (2018). Película biodegradable de almidón de maíz termoplástico y quitosano con actividad antimicrobiana empleada como envase activo. Matéria, 23(2), http://dx.doi.org/10.1590/s1517-707620180002.0423

Mali, S., \& Grossmann, M. V. E. (2003). Effects of yam starch films on storability and quality of fresh strawberries (Fragaria ananassa). Journal of Agricultural and Food Chemistry, 51(24), 7005-7011. PMid:14611162. http://dx.doi.org/10.1021/jf034241c

Mali, S., Grossmann, M. V. E., \& Yamashita, F. (2010). Starch films: production, properties and potential of utilization. Semina: Ciências Agrárias, 31(1), 137-156. http://dx.doi.org/10.5433/1679-0359.2010v31n1p137

Medina-Jaramillo, C., Ochoa-Yepes, O., Bernal, C., \& Famá, L. (2017). Active and smart biodegradable packaging based on starch and natural extracts. Carbohydrate Polymers, 176, 187-194. PMid:28927597.

http://dx.doi.org/10.1016/j.carbpol.2017.08.079

Moraes, A. R. F., Vidigall, M. C. T. R., Soares, N. F. F., Moraes, L. P., Mello, N. R., \& Gonçalves, M. P. J. (2011).

Desenvolvimento e avaliação de filme antimicrobiano aromatizado para aplicação em massa de pastel. Ciência Rural, 41(3), 537-543. http://dx.doi.org/10.1590/S0103-84782011000300029

Moro, T. M. A., Ascheri, J. L. R., Ortiz, J. A. R., Carvalho, C. W. P., \& Meléndez-Arévalo, A. (2017). Bioplastics of native starches reinforced with passion fruit peel. Food and Bioprocess Technology, 10(10), 1798-1808. http://dx.doi.org/10.1007/s11947-017-1944-x

Pająk, P., Przetaczek-Rożnowska, I., \& Juszczak, L. (2019). Development and physicochemical, thermal and mechanical properties of edible films based on pumpkin, lentil and quinoa starches. International Journal of Biological Macromolecules, 138, 441-449. PMid:31302126. http://dx.doi.org/10.1016/j.ijbiomac.2019.07.074

Pastor, C., Sánchez-González, L., Marcilla, A., Chiralt, A., Cháfer, M., \& González-Martínez, C. (2011). Quality and safety of table grapes coated with hydroxypropylmethylcellulose edible coatings containing propolis extract. Postharvest Biology and Technology, 60(1), 64-70. http://dx.doi.org/10.1016/j.postharvbio.2010.11.003

Rigo, L. N. (2006). Elaboração e caracterização de filmes comestíveis (Dissertação de mestrado). Universidade Regional Integrada do Alto do Uruguai e das Missões, Erechim.

Rocha, G. O., Farias, M. G., Carvalho, C. W. P., Ascheri, J. L. R., \& Galdeano, M. C. (2014). Filmes compostos biodegradáveis a base de amido de mandioca e proteína de soja. Polímeros, 24(5), 587-595. http://dx.doi.org/10.1590/0104-1428.1355

Rodríguez, M., Osés, J., Ziani, K., \& Maté, J. I. (2006). Combined effect of plasticizers and surfactants on the physical properties of starch based edible films. Food Research International, 39(8), 840-846. http://dx.doi.org/10.1016/j.foodres.2006.04.002

Roesler, R., Malta, L. G., Carrasco, L. C., Holanda, R. B., Sousa, C. A. S., \& Pastore, G. M. (2007). Atividade antioxidante de frutas do cerrado. Food Science and Technology, 27(1), 53-60. http://dx.doi.org/10.1590/S0101-20612007000100010

Sadeghizadeh-Yazdi, J., Habibi, M., Kamali, A. A., \& Banaei, M. (2019). Application of edible and biodegradable starch-based films in food packaging: a systematic review and meta-analysis. Current Research in Nutrition and Food Science Journal, 7(3), 624-637. http://dx.doi.org/10.12944/CRNFSJ.7.3.03 
Sapper, M., \& Chiralt, A. (2018). Starch-based coatings for preservation of fruits and vegetables. Coatings, 8(5), 152. http://dx.doi.org/10.3390/coatings8050152

Sari, P., Wijaya, C. H., Sajuthi, D., \& Supratman, U. (2012). Colour properties, stability, and free radical scavenging activity of jambolan (Syzygium cumini) fruit anthocyanins in a beverage model system: natural and co-pigmented anthocyanins. Food Chemistry, 132(4), 1908-1914. http://dx.doi.org/10.1016/j.foodchem.2011.12.025

Sessini, V., Arrieta, M. P., Kenny, J. M., \& Peponi, L. (2016). Processing of edible films based on nanoreinforced gelatinized starch. Polymer Degradation \& Stability, 132, 157-168. http://dx.doi.org/10.1016/j.polymdegradstab.2016.02.026

Shimazu, A. A., Mali, S., \& Grossmann, M. V. E. (2007). Efeitos plastificante e antiplastificante do glicerol e do sorbitol em filmes biodegradáveis de amido de mandioca. Semina: Ciências Agrárias, 28(1), 79-88. http://dx.doi.org/10.5433/16790359.2007v28n1p79

SobralP. J. A.MenegalliF. C.HubingerM. D.RoquesM. A.2001Mechanical, water vapor barrier and thermal properties of gelatin based edible films.Food Hydrocolloids15423432https://doi.org/10.1016/S0268-005X(01)00061-3

Sousa, M. S. B., Vieira, L. M., \& Lima, A. (2011). Fenólicos totais e capacidade antioxidante in vitro de resíduos de polpas de frutas tropicais. Brazilian Journal of Food Technology, 14(3), 202-210. http://dx.doi.org/10.4260/BJFT2011140300024

Souza, C. O., Silva, L. T., \& Druzian, J. I. (2012a). Estudo comparativo da caracterização de filmes biodegradáveis de amido de mandioca contendo polpas de manga e de acerola. Quimica Nova, 35(2), 262-267. http://dx.doi.org/10.1590/S010040422012000200006

Souza, S. M. A., Sobral, P. J. A., \& Menegalli, F. C. (2012b). Propriedades físicas de filmes comestíveis a base de proteínas miofibrilares de carne bovina. Semina: Ciências Agrárias, 33(1), 283-296. http://dx.doi.org/10.5433/1679-0359.2012v33n1p283

Talón, E., Vargas, M., Chiralt, A., \& González-Martínez, C. (2019). Antioxidant starch-based films with encapsulated eugenol: Application to sunflower oil preservation. Lebensmittel-Wissenschaft + Technologie, 113, 108290.

Veber, J., Petrini, L. A., Andrade, L. B., \& Siviero, J. (2015). Determinação dos compostos fenólicos e da capacidade antioxidante de extratos aquosos e etanólicos de Jambolão (Syzygium cumini L.). Revista Brasileira de Plantas Medicinais, 17(2), 267-273. http://dx.doi.org/10.1590/1983-084X/12_181

Vendramini, A. L., \& Trugo, L. C. (2000). Chemical composition of acerola fruit (Malpighia punicifolia L.) at three stages of maturity. Food Chemistry, 71(2), 195-198. http://dx.doi.org/10.1016/S0308-8146(00)00152-7

Vicentini, N. M. (2003). Elaboração e caracterização de filmes comestíveis à base de fécula de mandioca para uso em póscolheita. (Tese de Doutorado). Universidade Estadual Paulista, Botucatu.

Wang, L. F., \& Rhim, J. W. (2016). Grapefruit seed extract incorporated antimicrobial LDPE and PLA films: effect of type of polymer matrix. Lebensmittel-Wissenschaft + Technologie, 74, 338-345. http://dx.doi.org/10.1016/j.lwt.2016.07.066

Wrona, M., Nerín, C., Alfonso, M. J., \& Caballero, M. Á. (2017). Antioxidant packaging with encapsulated green tea for fresh minced meat. Innov. Food Sci. Emerg, 41, 307-313. http://dx.doi.org/10.1016/j.ifset.2017.04.001

Zhang, Z. J., Li, N., Li, H. Z., Li, X. J., Cao, J. M., Zhang, G. P., \& He, D. L. (2018). Preparation and characterization of biocomposite chitosan film containing Perilla frutescens (L.) Britt. essential oil. Industrial Crops and Products, 112, 660-667. http://dx.doi.org/10.1016/j.indcrop.2017.12.073

Financiamento: Nenhum. 